Mens

Revue d'histoire intellectuelle et culturelle

\title{
Mise au point sur un texte de Jacques Rouillard
}

\section{Gérard Bouchard}

Volume 13, numéro 1, automne 2012

S’approprier le passé des autres : les usages de l'histoire internationale au Québec avant la Révolution tranquille

URI : https://id.erudit.org/iderudit/1019700ar

DOI : https://doi.org/10.7202/1019700ar

Aller au sommaire du numéro

Éditeur(s)

Centre de recherche en civilisation canadienne-française

ISSN

1492-8647 (imprimé)

1927-9299 (numérique)

Découvrir la revue

Citer ce document

Bouchard, G. (2012). Mise au point sur un texte de Jacques Rouillard. Mens,

13(1), 109-112. https://doi.org/10.7202/1019700ar d'utilisation que vous pouvez consulter en ligne.

https://apropos.erudit.org/fr/usagers/politique-dutilisation/ 


\title{
Répliques
}

\section{Mise au point sur un texte de Jacques Rouillard}

\author{
Gérard Bouchard \\ Université du Québec à Chicoutimi
}

Dans un numéro récent de la revue Mens (printemps 2012, p. 145-163), Jacques Rouillard me prend à partie sur deux points : a) je rejetterais le courant historiographique québécois couramment qualifié de révisionniste et b) je me serais contredit dans mes travaux, d'un côté en vantant le dynamisme de la société canadienne-française (avant la Révolution tranquille) et, de l'autre, en dénonçant son immobilisme et son impuissance. En somme, je serais à la fois un critique et un défenseur de la thèse de la Grande Noirceur. Ma réplique peut se résumer en quelques mots : le texte de mon collègue donne dans une polarisation à outrance et montre ainsi un étonnant manque de nuance, qui est la source d'une grande confusion et de contradictions élémentaires, en plus de fermer la voie à une meilleure compréhension de phénomènes complexes.

À propos de l'historiographie " révisionniste " - que je préfere appeler «moderniste " - j’ai toujours écrit qu'elle avait apporté une correction salutaire à la vision de notre passé en le projetant dans toute sa diversité et sa complexité. J'ai toujours loué également sa pertinence ainsi que la qualité et l'ampleur de son apport (j'aurais été mal venu de faire autrement, mes propres travaux étant souvent rangés sous cette étiquette). Cela dit, j'en ai souligné aussi certains 
excès chez quelques auteurs (de ce côté-là également règnent la diversité et la complexité; l'histoire moderniste n'est pas une Église, on n'y trouve pas d'orthodoxie). J'ai aussi fait valoir qu'après quelques décennies marquées par la publication d'ouvrages remarquables, ce courant avait un peu épuisé son potentiel de renouvellement. En d'autres mots, son succès et son héritage sont incontestables. Chacun peut constater qu'il a pleinement atteint ses objectifs, ses principales propositions faisant maintenant partie de la vulgate scientifique. Affirmer qu'un courant historiographique a "accompli son programme », est-ce le dénigrer ou souligner sa réussite?

Je me félicite personnellement de ce succès et me réjouis de ce que quelques ouvrages pionniers resteront longtemps des références (dans mon esprit, les travaux de Jacques Rouillard sur le syndicalisme sont de ceux-là). Mais, tout en nous appuyant sur les acquis, il est temps de continuer l'exploration de notre passé en donnant la priorité à d'autres angles prometteurs, en accord avec d'autres questions pressantes qui se posent au présent et que la science historique peut éclairer. Je songe, parmi d'autres, aux fondements symboliques de l'intégration et de la cohésion sociale (et de son contraire : les divisions, les conflits, les exclusions de toutes sortes), à la thématique de la diversité ethnoculturelle ou à tout ce qui concerne le rapport au monde. Encore une fois, ce faisant, il n'est pas question de rejeter les thèses modernistes car elles font partie des acquis, bien qu'inévitablement la recherche à venir conduira à les nuancer - peut-être substantiellement sur certains points.

À propos de mes travaux qui, selon Jacques Rouillard, réfuteraient la Grande Noirceur en même temps qu'ils l'accréditeraient, il y aurait beaucoup à dire et je ne manquerai pas de le faire à une prochaine occasion. Je m'en tiendrai pour le moment à quelques remarques.

D'abord, on voit dans cette critique un effet de la polarisation excessive que je déplorais plus haut. Par exemple, je suis convaincu de l'importance du courant libéral dans l'histoire du Québec, je n'ai jamais présenté le conservatisme clérical comme étant sa " composante exclusive " et je rejette la thèse du " monolithisme idéologique ". 
Mais, en même temps, j'ai toujours pensé que le passé québécois montrait des traits, des stigmates qui lui conféraient un caractère distinctif en Occident (incluant le Nouveau Monde), notamment à cause de son statut minoritaire et de sa longue soumission au joug colonial.

D'autre part, des distinctions importantes font ici défaut. Dans mon article paru dans Recherches sociographiques (septembre-décembre 2005), je m'employais à montrer que de nombreuses tendances ou épisodes associés à la Grande Noirceur québécoise se retrouvaient également ailleurs en Occident ${ }^{1}$. Je rappelais aussi que plusieurs changements imputés à la décennie 1960 avaient pris naissance bien avant. Ces deux mises au point visaient à relativiser la vision de la Grande Noirceur, sans nier la grande effervescence des années 1960 et les importants changements qu'elles ont apportés au Québec.

Jacques Rouillard voit là une contradiction; j’y vois plutôt un souci de faire (pièces à l'appui) la part des choses, ce qui est une règle fondamentale du métier d'historien. La même règle invite aussi à démêler, dans les visions du passé qui sont proposées dans le débat public, ce qui relève de l'idéologie d'un groupe ou d'une classe sociale en quête de reconnaissance et de promotion, et ce qui procède de l'analyse scientifique bien documentée.

Ce qui précède me mettrait en contradiction avec les conclusions de mes études sur l'évolution de la pensée canadienne-française, à savoir que cette pensée était en butte à des contradictions fondamentales qu' elle n'est pas arrivée à surmonter, d'où un sentiment d'impuissance. Je précise d'abord que je n'ai pas introduit cette thématique de l'impuissance, plusieurs auteurs l'ayant abordée avant (et après) moi. L'un des intellectuels qui l'ont le plus martelée est Pierre Vadeboncoeur, qui fut justement l'un des principaux artisans de la Révolution tranquille. Ce que Jacques Rouillard passe sous silence, c'est que dans ces travaux, j’analyse essentiellement la pensée nationale, plus précisé-

${ }^{1}$ Il en va de même pour bien des changements associés à la Révolution tranquille. Tout cela illustre simplement le fait que le Québec fait partie de l'Occident et que son devenir en est inséparable. 
ment les efforts de divers intellectuels pour faire advenir la nation. Les élites que j'ai étudiées sont celles qui ont essayé de concrétiser le rêve national d'émancipation en mettant de l'avant des définitions, des orientations politiques, des visions de l'avenir et des stratégies pour les réaliser. Et je me suis autorisé à parler d'échec en mettant simplement en rapport les aspirations, les objectifs annoncés au départ (par exemple, l'autonomie ou la souveraineté de la nation) et les résultats concrets de l'action.

En parallèle, dans plusieurs secteurs de la société, la vie pouvait continuer : la croissance démographique, la colonisation, l'urbanisation, l'industrialisation, et le reste. Mais ce sont d'autres moteurs et d'autres acteurs qui prenaient en charge ces mouvements : les familles, les grands capitalistes (en majorité étrangers), les commerçants et petits entrepreneurs, les politiciens souvent, les travailleurs... C'est justement l'un des traits remarquables de l'ancienne pensée nationaliste - en même temps que l'une des sources de son impuissance - que d'avoir été largement décrochée de ces mouvements, que de s'être déployée à distance et souvent en contradiction avec la réalité en marche. J'ai parlé à ce propos de désarticulation, pour désigner tout un courant de pensée qui, à cause de contradictions non résolues ${ }^{2}$, n'arrive pas à se mettre en prise sur le devenir de la société. Résultat, il tend à inhiber ce devenir plutôt qu'à le propulser. Au Québec, le chanoine Groulx est le plus éminent (et le plus tragique) représentant de cette impasse.

Ce que nous enseignent surtout les critiques de Jacques Rouillard, c'est que la simplification est parfois ennemie de la clarté et que la polarisation trahit souvent la réalité. Je le déplore d'autant plus qu'il a lui-même contribué magistralement à faire la lumière sur une dimension très importante de notre passé - ce pour quoi je lui garde toute mon admiration et mon respect.

2 Écartons ici un malentendu : une contradiction non résolue, c'est soit une incohérence qu'on n'arrive pas à supprimer, soit une incohérence qu'on n'arrive pas à harnacher - par exemple, en recourant à un mythe. En lui-même, le contradictoire n'est donc pas nécessairement un facteur d'empêchement. 